\title{
Investigating the relationship between COVID-19- related and distress and ICD-11 adjustment disorder: two cross-sectional studies
}

\author{
Menachem Ben-Ezra, Wai Kai Hou and Robin Goodwin
}

\section{Background}

To assess the prevalence of elevated risk of serious mental illness and probable ICD-11 adjustment disorder in the UK population at two time points during COVID-19, and their association with COVID-19-related stressful events.

\section{Aims}

To check the dose-response model for stress between the number of COVID-19-related stressful events and mental health indices.

\section{Method}

We conducted two cross-sectional studies, using internet survey samples across the UK ( $N=1293$ for study 1; $N=1073$ for study 2). Samples used internet panel surveys during March-April 2020 and 3 months later (June 2020), and used random stratified samples. Studies assessed prevalence of serious risk of mental illness and probable ICD-11 adjustment disorder.

\section{Results}

Elevated risk of serious mental illness was found among those with COVID-19-related social life or occupationally stressful events (study 1). Elevated risk of serious mental illness and probable ICD-11 adjustment disorder was evident among those reporting COVID-19-related stressful events (personal health problems and caregiving; study 2). Cumulative COVID-19-related stressful events were associated with elevated risk of serious mental illness in study 1 (odds ratio 1.65; 95\% Cl 1.03-2.64; $P=0.037$ ), and with both elevated risk of serious mental illness (odds ratio 2.19; 95\% $\mathrm{Cl} 1.15-4.15 ; P=0.017$ ) and probable ICD-11 adjustment disorder (odds ratio $2.45 ; 95 \% \mathrm{Cl} 1.27-4.72 ; P=0.007$ ) in study 2.

\section{Conclusions}

Psychiatrists should be aware that COVID-19-related stressful events can lead to serious psychological problems. Mental health professionals need to pay particular attention to patients who report cumulative COVID-19-related stressful events, and consider them for mental health assessment and treatment.

\section{Keywords}

COVID-19; serious mental illness; ICD-11; adjustment disorder; health.

\section{Copyright and usage}

(c) The Author(s), 2020. Published by Cambridge University Press on behalf of the Royal College of Psychiatrists. This is an Open Access article, distributed under the terms of the Creative Commons Attribution licence (http://creativecommons.org/ licenses/by/4.0/), which permits unrestricted re-use, distribution, and reproduction in any medium, provided the original work is properly cited.
SARS-CoV-2, the virus that causes COVID-19, first emerged in the UK in January 2020. ${ }^{1}$ The UK government closed schools on 20 March 2020, and imposed a nationwide lockdown on 23 March $2020 .^{2}$ This lockdown immediately generated COVID-19-related stressful events, including stressors that were social (restricted face-to-face interactions, including with intimate and familial relations), occupational (unemployment, furlough, reduced hours, working from home) and educational (cancelled courses, changed schedules, remote studying). In addition, infection outbreaks led to a number of individuals being placed in quarantine. A recent Chinese study showed that quarantine restricted every aspect of life and increased mental burden. ${ }^{3}$ Although isolation restricts freedom of movement, other restrictions in COVID-19-related stressful events may stem from different sources. According to the conservation of resources theory ${ }^{4}$ and related frameworks, ${ }^{5}$ these major disruptions in daily lives can provoke psychological distress. During the COVID-19 pandemic, individuals may experience loss of those resources normally used to effectively cope with, and manage stressful situations. We suggest that this threat is cumulative across COVID-19-related stressful events, with the overwhelming effect a psychological version of a 'cytokine storm', an immune system failure with serious psychological implications.

We examine associations among COVID-19-related stressful events, underlying health conditions associated with increased mortality from COVID-19, being in isolation and serious mental illness. In addition, we hypothesise that a cumulative loss of resources will be associated with elevated risk of serious mental illness, with the larger the number of events generated by COVID-19, the greater the mental toll. We collect national cross-sectional data at two time points: the first survey during the peak of the pandemic (average new daily cases 3206, with 2078 new deaths), and the second data wave as the pandemic plateaued (average new daily cases 1042, with 137 new deaths; Fig. 1). Understanding the differential effects of disruption caused by COVID-19 during two very different periods should provide clinicians with a better understanding of its negative effect on mental health outcomes over the life course of the pandemic.

\section{Method}

All procedures performed in studies involving human participants were in accordance with the ethical standards of the Ethics Committee of Ariel University (approval number AU-SOC-MBE20200225), and with the 1964 Helsinki Declaration and its later amendments or comparable ethical standards. Each participant signed an electronic informed consent form.

\section{Study 1}

Sampling

We conducted a sample of the UK population by using an internet panel $(N=1293)$, with a random and stratified sampling. Participants were recruited between 30 March and 2 April 2020 (i.e. 1 week after the UK national lockdown). The mean age of the participants was 51.51 years (s.d. 14.75, range 18-75), 53.3\% were 


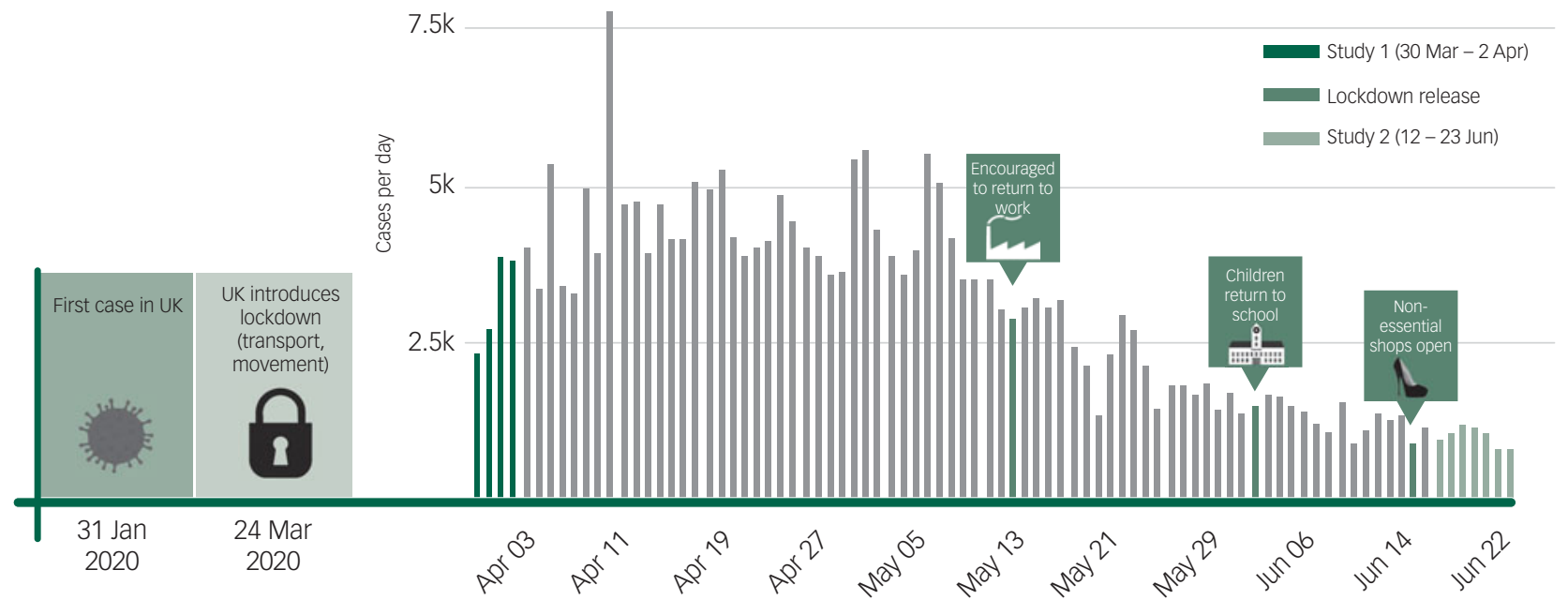

Fig. 1 Data collection against UK pandemic timeline.

Infographic was provided by David Goodwin based on figures from https://www.worldometers.info/.

female $(n=689), 69.0 \%$ reported being in a committed relationship $(n=892)$ and $27.2 \%(n=352)$ reported having a background medical condition (hypertension, diabetes, cardiovascular disease, chronic respiratory disease, chronic obstructive pulmonary disease and cancer). The response rate was 37\%. See Table 1 for more information.

\section{Measurements}

Being in isolation was measured by the item: 'Are you currently in quarantine because of the coronavirus?' ( 0 for no, 1 for yes).

COVID-19-related stressful events were measured by three questions: 'In the past month, has the COVID-19 pandemic affected your relationships or social life?', 'In the past month, has the COVID-19 pandemic affected your work or ability to work?' and 'In the past month, has the COVID-19 pandemic affected your school or college work?'. Each question was rated on a scale with response categories of 0 for not at all, 1 for slightly, 2 for moderately, 3 for very much and 4 for extremely. Each question was aggregated based on the two highest categories versus the rest (low disruption of COVID-19-related stressful events for categories 0-2 versus high disruption in COVID-19-related stressful events for categories 3-4). In addition, we created a severity index of $0-3$ to indicate the number of COVID-19-related stressful events.

\section{Outcome variable}

Screening for serious mental illness was measured using the six-item K6 scale, ${ }^{6}$ with direct reference to COVID-19 pandemic in the instructions. Items included: 'During the past 30 days, how often did you feel: 1. nervous; 2. hopeless; 3 . restless or fidgety; 4 . so depressed that nothing could cheer you up; 5 . everything was an effort; 6. worthless?'. Each category was rated as one of following: 0 , none of the time; 1 , a little of the time; 2 , some of the time; 3 , most of the time; or 4, all of the time. Scores ranged from 0 to 24, with $\geq 13$ indicating elevated risk of serious mental illness. ${ }^{6}$ Cronbach $\alpha$ was satisfactory $(0.90)$.

\section{Statistical analyses}

A multivariate logistic regression with elevated risk of serious mental illness (K6 score $\geq 13$ ) as the outcome measure entered the following variables: demographics (age, gender, marital status, background illness, isolation) and three COVID-19-related stressful events. A second logistic regression also included an index for cumulative COVID-19-related stressful events (ranging from zero to three) instead of each single event, as in the former logistic regression. Each category in the index was compared with the reference group (no COVID-19-related stressful events). For each variable, we calculated the odds ratio and 95\% confidence interval, using SPSS version 25 for Windows (IBM).

\section{Study 2}

The above study included only a limited number of COVID-19related stressful events, and so did not cover a number of major aspects of life. In our second study, we aimed to replicate the findings of study 1, using a different national sample and an expanded list of stressful events. We collected data as the pandemic's first wave in the UK plateaued and the four constituent countries moved out of lockdown.

In this second study, we considered an additional aspect of the mental health toll of the COVID-19 pandemic: ICD-11 adjustment disorder. Contrary to serious mental illness, a broad concept based on multiple constructs mainly drawing on depression and anxiety, ${ }^{6}$ the ICD-11 adjustment disorder is specifically associated with stress, providing a distinct mental disorder (adjustment disorder: code $6 \mathrm{~B} 43$ in ICD-11) alongside a specific narrative description to provide and guide specific diagnosis. ${ }^{7}$

Based on study 1, we hypothesised that COVID-19-related stressful events will be associated with elevated risk of serious mental illness and probable ICD-11 adjustment disorder. Based on a dose-response model, ${ }^{7}$ we predicted that the more stressful events a person experiences, the higher the likelihood that they will exhibit elevated risk of serious mental illness and ICD-11 adjustment disorder.

\section{Sampling}

We conducted a sample of the UK population, using an internet panel $(N=1073)$, with random stratified sampling. Participants were recruited between 17 and 23 June 2020. The mean age of the participants was 54.36 years (s.d. 12.14, range 20-75), 55.1\% were female $(n=591), 70.5 \%$ reported being in a committed relationship $(n=757)$ and $28.6 \%(n=307)$ reported having a background medical condition (hypertension, diabetes, cardiovascular disease, chronic respiratory disease, chronic obstructive pulmonary disease and cancer). The response rate was $42 \%$. See Table 1 for more information. 


\begin{tabular}{|c|c|c|c|c|c|c|c|c|}
\hline \multirow[b]{2}{*}{ Variables } & \multicolumn{4}{|c|}{ Study 1} & \multicolumn{4}{|c|}{ Study 2} \\
\hline & Mean & s.d. & N & $\%$ & Mean & s.d. & N & $\%$ \\
\hline \multicolumn{9}{|l|}{ Demographics } \\
\hline Age, years & 51.51 & 14.75 & & & 54.36 & 12.14 & & \\
\hline Gender, female & & & 689 & 53.3 & & & 591 & 55.1 \\
\hline Being in a committed relationship, yes & & & 892 & 69.0 & & & 757 & 70.5 \\
\hline Being in risk group for COVID-19, yes & & & 352 & 27.2 & & & 307 & 28.6 \\
\hline \multicolumn{9}{|l|}{ Lockdown } \\
\hline Currently in isolation, yes & & & 389 & 30.1 & & & 124 & 11.6 \\
\hline \multicolumn{9}{|l|}{ Disrupted COVID-19-related stressful events } \\
\hline High disruption in social domain, yes & & & 547 & 42.3 & & & & \\
\hline High disruption in occupational domain, yes & & & 442 & 34.2 & & & & \\
\hline High disruption in educational domain, yes & & & 485 & 37.5 & & & & \\
\hline \multicolumn{9}{|l|}{ Index of disrupted COVID-19-related stressful events } \\
\hline No disruption in COVID-19-related stressful eventsa & & & 513 & 39.7 & & & & \\
\hline One disruption in COVID-19-related stressful events & & & 315 & 24.4 & & & & \\
\hline Two disruptions in COVID-19-related stressful events & & & 236 & 18.3 & & & & \\
\hline Three disruptions in COVID-19-related stressful events & & & 229 & 17.7 & & & & \\
\hline \multicolumn{9}{|l|}{ COVID-19-related stressful events } \\
\hline Financial problems, yes & & & & & & & 217 & 20.2 \\
\hline Work problems, yes & & & & & & & 177 & 16.5 \\
\hline Educational problems, yes & & & & & & & 41 & 3.8 \\
\hline Housing problems, yes & & & & & & & 61 & 5.7 \\
\hline Relationship problems, yes & & & & & & & 140 & 13.0 \\
\hline Personal health problems, yes & & & & & & & 321 & 29.9 \\
\hline A loved one's health problems, yes & & & & & & & 303 & 28.8 \\
\hline Caregiving problems, yes & & & & & & & 190 & 17.7 \\
\hline \multicolumn{9}{|l|}{ Index of CovID-19-related stressful events } \\
\hline No eventsa & & & & & & & 486 & 45.3 \\
\hline One event & & & & & & & 193 & 18.0 \\
\hline Two events & & & & & & & 170 & 15.8 \\
\hline Three events & & & & & & & 101 & 9.4 \\
\hline Four or more events & & & & & & & 123 & 11.5 \\
\hline \multicolumn{9}{|l|}{ Outcome variables } \\
\hline Elevated serious mental illness $(K 6 \geq 13)$ & & & 215 & 16.6 & & & 153 & 14.3 \\
\hline Probable ICD-11 adjustment disordera & & & & & & & 171 & 15.9 \\
\hline
\end{tabular}

\section{Measurements}

We used similar measures as in study 1 , with the following exceptions. COVID-19-related stressful events were measured by the International Adjustment Disorder Questionnaire (IADQ). ${ }^{7}$ The IADQ comprises two parts. First is a COVID-19-modified checklist of stressful events (stressors list) with eight events: financial problems (e.g. difficulty paying bills, being in debt); work problems (e.g. unemployment, redundancy, retirement, problems/conflicts with colleagues, change of job role); educational problems (e.g. difficulty with course work, deadline pressure); housing problems (e.g. stressful home move, difficulty finding a secure residence, lack of secure residence); relationship problems (e.g. break-up, separation or divorce, conflict with family or friends, intimacy problems); personal health problems (e.g. illness onset or deterioration, medication issues, injury or disability); a loved one's health problems (e.g. illness onset or deterioration, medication issues, injury or disability) and caregiving problems (e.g. emotional stress, time demands). Participants checked 'yes' for each event applicable to them, creating a cumulative score categorised as follows: 0 , zero events; 1 , one event; 2 , two events; 3 , three events and 4 , four or more events (the number of participants reporting more than four events was $67(6.1 \%))$. The second IADQ component assesses adjustment disorder core symptoms, duration and functional impairment. The first six questions measure two symptoms clusters ('preoccupation' and 'failure to adapt'). Three subsequent questions examine functional impairment in life domains (social, occupational (educational) and other domains of life). These nine questions were rated as follows: 0 , not at all; 1 , a little bit; 2, moderately; 3 , quite a bit and 4, extremely. The tenth question assesses duration of symptoms (coded as 0 for no and 1 for yes).

The algorithm for a probable diagnosis of ICD-11 adjustment disorder requires the presence of a psychosocial stressor (score $\geq 1$ on the IADQ stressor list), at least one preoccupation symptom rated $\geq 2$, at least one failure-to-adapt symptom rated $\geq 2$, and evidence of functional impairment rated $\geq 2$. $^{7}$ Cronbach $a$ was satisfactory for IADQ (0.97).

\section{Outcome variables}

To limit the tautological risk of using the stressor list as both an independent and dependent variable, we computed a second, modified algorithm, which excludes the IADQ stressor list.

To use the second part of the IADQ (adjustment disorder scale) as an outcome measure, we viewed the COVID-19 pandemic as a global stressor, thus satisfying the criteria for exposure to a stressful event. We used a slightly modified algorithm to prevent a tautology in this study, utilising the stressor list as part as the dependent and independent variables (measuring exposure to COVID-19-related stressful events as the independent variable and as part of the dependent variable). The modified algorithm used in the study is identical to algorithm presented above excluding the IADQ stressor list.

The results section includes the prevalence of probable ICD-11 adjustment disorder among the UK population according to the IADQ original algorithm, and the prevalence of ICD-11 adjustment disorder when using the modified algorithm. 
Table 2 Logistic regressions in the UK sample from March-April 2020, for the association of the study variables with serious mental illness (study 1 $N=1293)$

Elevated risk of serious mental illness ( $\mathrm{K} 6$ score $\geq 13$ )

\begin{tabular}{|c|c|c|c|c|}
\hline Variables & Adjusted odds ratio $(95 \% \mathrm{Cl})$ & $P$-value & Adjusted odds ratio $(95 \% \mathrm{Cl})$ & $P$-value \\
\hline \multicolumn{5}{|l|}{ Demographics } \\
\hline Age, years & $0.98(0.96-0.99)$ & $<0.001$ & $0.98(0.97-0.99)$ & $<0.001$ \\
\hline Gender, female & $1.38(1.00-1.90)$ & 0.049 & $1.39(1.01-1.91)$ & 0.045 \\
\hline Being in a committed relationship, yes & $1.15(0.82-1.61)$ & 0.425 & $1.19(0.85-1.67)$ & 0.319 \\
\hline Being in risk group for COVID-19, yes & $1.30(0.90-1.89)$ & 0.166 & $1.29(0.90-1.87)$ & 0.186 \\
\hline \multicolumn{5}{|l|}{ Lockdown } \\
\hline Currently in isolation, yes & $1.69(1.21-2.34)$ & 0.002 & $1.72(1.24-2.40)$ & 0.001 \\
\hline \multicolumn{5}{|l|}{ Disrupted COVID-19-related stressful events } \\
\hline High disruption in social domain, yes & $2.59(1.81-3.71)$ & $<0.001$ & & \\
\hline High disruption in occupational domain, yes & $1.53(1.06-2.21)$ & 0.022 & & \\
\hline High disruption in educational domain, yes & $1.37(0.95-1.98)$ & 0.092 & & \\
\hline \multicolumn{5}{|l|}{$\begin{array}{l}\text { Index of disrupted COVID-19-related stressful events } \\
\text { No disruption in COVID-19-related stressful events }\end{array}$} \\
\hline One disruption in COVID-19-related stressful events & & & $1.65(1.03-2.64)$ & 0.037 \\
\hline Two disruptions in COVID-19-related stressful events & & & $2.43(1.53-3.87)$ & $<0.001$ \\
\hline Three disruptions in COVID-19-related stressful events & & & $5.56(3.60-8.60)$ & $<0.001$ \\
\hline
\end{tabular}

\section{Serious mental illness}

Screening for serious mental illness ${ }^{6}$ was measured in the same way as in study 1 . Cronbach a was satisfactory for K6 (0.92).

\section{Statistical analysis}

A multivariate logistic regression used elevated risk of serious mental illness (K6 score $\geq 13$ ) as the outcome measure, with the following variables entering the equation: demographics (age, gender, marital status, background illness, isolation) and exposure to COVID-19-related stressful event (with all events entered simultaneously). The second logistic regression was the same, except that we used an index for cumulative COVID-19-related stressful events (ranging from zero to eight) instead of the eight single events. Each category in the index was compared with the reference group (zero COVID-19-related stressful events). The same method was used, with probable ICD-11 adjustment disorder as the outcome variable (based on the modified algorithm). For each variable, we calculated the odds ratio and $95 \%$ confidence interval, and conducted a comparison of proportions, comparing the percentage of those with elevated risk of serious mental illness in each sample (independent sample comparison). Finally, we compared the models associated with elevated psychological distress with the modified algorithm for ICD-11 probable adjustment disorder, using a chi-square test.

To test the dose-response model for stress, we compared the association of each stressful event and the total events score with elevated risk of serious mental illness and modified algorithm for ICD-11 probable adjustment disorder, respectively, using Steiger's $z$-test. ${ }^{8}$ Data were analysed with SPSS version 25 for Windows (IBM).

\section{Results}

For study 1, elevated risk of serious mental illness was found in $16.6 \%$ of the sample $(n=215)$. Elevated risk of serious mental illness was associated with disruption in social (odds ratio 2.59; 95\% CI 1.81-3.71; $P<0.001$ ) and occupational domains (odds ratio $1.53 ; 95 \%$ CI $1.06-2.21 ; P=0.022$ ). (Table 1). Elevated risk of serious mental illness was associated with cumulative COVID19-related stressful events: odds ratio of 1.65 for one COVID-19related stressful event (95\% CI 1.03-2.64; $P=0.037$ ), 2.43 for two
COVID-19-related stressful events (95\% CI 1.53-3.87; $P<0.001)$ and 5.56 for three COVID-19-related stressful events (95\% CI 3.60-8.60; $P<0.001$ ) (Table 2).

The results of study 2 showed that elevated risk of serious mental illness was found in $14.3 \%$ of the sample $(n=153)$. The proportion comparison between the two samples indicated that elevated risk of serious mental illness was not significant (difference of $2.3 \%$; $95 \%$ CI $-0.64 \%$ to $5.20 \% ; \chi^{2}=2.360$; d.f. $1 ; P=0.125$ ).

Probable ICD-11 adjustment disorder according to the IADQ algorithm was found among $15.9 \%$ of the sample $(n=171)$, and probable ICD-11 adjustment disorder according to the IADQ modified algorithm was found among $17.8 \%$ of the sample $(n=191)$.

Elevated risk of serious mental illness was significantly associated with housing problems (odds ratio 2.71; 95\% CI 1.39-5.30; $P=$ 0.003 ), the participant's own health problems (odds ratio 3.43; $95 \%$ CI $2.20-5.30 ; P<0.001$ ) and caregiving problems (odds ratio 1.83; 95\% CI 1.14-2.94; $P=0.013$ ). Elevated risk of serious mental illness was significantly associated with cumulative COVID-19related stressful events compared with those participants reporting no COVID-19-related stressful events. These results were significant for those who had experienced at least one COVID-19-related stressful event (odds ratio 2.19; 95\% CI 1.15-4.15; $P=0.017$ ), with an increased likelihood of elevated risk of serious mental illness for those reporting experiencing four or more COVID-19-related stressful events (odds ratio 12.41; 95\% CI 7.05-21.84; $P<0.001$ ). In addition, being younger was associated with elevated risk of serious mental illness for both measures. For more information, see Table 3. These results echoed the results of study 1 .

A similar pattern emerged with regard to probable ICD-11 adjustment disorder according to the modified algorithm. Probable ICD-11 adjustment disorder was significantly associated with the participant's own health problems (odds ratio 2.07; 95\% CI $1.38-3.10 ; P<0.001)$, the participant's loved one's health problems (odds ratio $1.85 ; 95 \%$ CI 1.22-2.80; $P=0.004$ ) and caregiving problems (odds ratio 2.59; 95\% CI 1.68-3.99; $P<0.001$ ). Using the modified algorithm, probable ICD-11 adjustment disorder was significantly associated with cumulative COVID-19-related stressful events compared with those participants reporting no COVID-19related stressful events. These results were significant for those who had experienced at least one COVID-19-related stressful event (odds ratio $2.45 ; 95 \%$ CI $1.27-4.72 ; P=0.007$ ), with an increased likelihood of probable ICD-11 adjustment disorder for those reporting experiencing four or more COVID-19-related 
Table 3 Logistic regressions in the UK sample from June 2020, for the association of the study variables with serious mental illness (study 2; $N=1073$ )

\begin{tabular}{|c|c|c|c|c|}
\hline \multirow[b]{2}{*}{ Variables } & \multicolumn{4}{|c|}{ Elevated risk of serious mental illness ( $\mathrm{K} 6$ score $\geq 13$ ) } \\
\hline & Adjusted odds ratio $(95 \% \mathrm{Cl})$ & $P$-value & Adjusted odds ratio $(95 \% \mathrm{Cl})$ & $P$-value \\
\hline \multicolumn{5}{|l|}{ Demographics } \\
\hline Age, years & $0.96(0.95-0.98)$ & $<0.001$ & $0.97(0.96-0.99)$ & $<0.001$ \\
\hline Gender, Female & $1.45(0.97-2.15)$ & 0.069 & $1.33(0.90-1.97)$ & 0.146 \\
\hline Being in a committed relationship, yes & $1.39(0.92-2.09)$ & 0.114 & $1.42(0.95-2.13)$ & 0.090 \\
\hline Being in risk group for COVID-19, yes & $1.14(0.73-1.77)$ & 0.575 & $1.42(0.94-2.16)$ & 0.098 \\
\hline \multicolumn{5}{|l|}{ Lockdown } \\
\hline Currently in isolation, yes & $1.62(0.96-2.73)$ & 0.068 & $1.54(0.91-2.59)$ & 0.107 \\
\hline \multicolumn{5}{|l|}{ COVID-19-related stressful events, yes } \\
\hline Financial problems & $1.45(0.92-2.30)$ & 0.113 & & \\
\hline Work problems & $0.86(0.52-1.45)$ & 0.581 & & \\
\hline Educational problems & $0.82(0.36-1.89)$ & 0.643 & & \\
\hline Housing problems & $2.71(1.39-5.30)$ & 0.003 & & \\
\hline Relationship problems & $1.42(0.86-2.33)$ & 0.174 & & \\
\hline Personal health problems & $3.43(2.20-5.33)$ & $<0.001$ & & \\
\hline A loved one's health problems & $1.29(0.82-2.04)$ & 0.269 & & \\
\hline Caregiving problems & $1.83(1.14-2.94)$ & 0.013 & & \\
\hline \multicolumn{5}{|l|}{$\begin{array}{l}\text { Index of COVID-19-related stressful events } \\
\text { No events }\end{array}$} \\
\hline One event & & & $2.19(1.15-4.15)$ & 0.017 \\
\hline Two events & & & $3.68(2.03-6.65)$ & $<0.001$ \\
\hline Three events & & & $6.98(3.78-12.90)$ & $<0.001$ \\
\hline Four or more events & & & $12.41(7.05-21.84)$ & $<0.001$ \\
\hline
\end{tabular}

stressful events (odds ratio 17.81; 95\% CI 9.92-31.97; $P<0.001$ ). In addition, being younger and currently being in isolation were associated with elevated serious mental illness for both measures. For more information, see Table 4.

In addition, we tested the models for association with the aforementioned (independent) variables examining the elevated risk of serious mental illness and the modified algorithm for probable ICD-11 adjustment disorder. The $-2 \log$ likelihood in the first model (Table 3) associated with elevated risk of serious mental illness was 723.272. This compares with 828.809 for the $-2 \log$ likelihood for the model for probable ICD-11 adjustment disorder (Table 4). The results of the chi-square test were significant at $P<0.005$. Hence, there was better model fit for the elevated risk of serious mental illness.

Finally, we tested the dose-response model for elevated risk for serious mental illness. A Steiger's $z$-score of 1.609 indicates that the dose-response model was not significant for elevated risk of serious mental illness. However, the modified algorithm for probable ICD-11 adjustment disorder showed a significant Steiger's $z$-score of 2.375, indicating a dose-response association.

\begin{tabular}{|c|c|c|c|c|}
\hline \multirow[b]{2}{*}{ Variables } & \multicolumn{4}{|c|}{ ICD-11 probable adjustment disorder (modified algorithm) } \\
\hline & Adjusted odds ratio $(95 \% \mathrm{Cl})$ & $P$-value & Adjusted odds ratio $(95 \% \mathrm{Cl})$ & $P$-value \\
\hline \multicolumn{5}{|l|}{ Demographics } \\
\hline Age, years & $0.90(0.97-1.00)$ & 0.080 & $0.99(0.099-1.02)$ & 0.092 \\
\hline Gender, female & $1.13(0.79-1.62)$ & 0.510 & $1.01(0.70-1.49)$ & 0.963 \\
\hline Being in a committed relationship, yes & $1.43(0.98-2.09)$ & 0.065 & $1.21(0.82-1.77)$ & 0.336 \\
\hline Being in risk group for COVID-19, yes & $0.90(0.59-1.37)$ & 0.631 & $0.96(0.64-1.43)$ & 0.825 \\
\hline \multicolumn{5}{|l|}{ Lockdown } \\
\hline Currently in isolation, yes & $1.89(1.16-3.06)$ & 0.010 & $1.90(1.16-3.10)$ & 0.011 \\
\hline \multicolumn{5}{|l|}{ COVID-19-related stressful events } \\
\hline Financial problems, yes & $1.48(0.96-2.27)$ & 0.076 & & \\
\hline Work problems, yes & $1.37(0.86-2.19)$ & 0.187 & & \\
\hline Educational problems, yes & $1.60(0.71-3.59)$ & 0.253 & & \\
\hline Housing problems, yes & $1.39(0.71-2.73)$ & 0.335 & & \\
\hline Relationship problems, yes & $1.32(0.82-2.11)$ & 0.249 & & \\
\hline Personal health problems, yes & $2.07(1.38-3.10)$ & $<0.001$ & & \\
\hline A loved one's health problems, yes & $1.85(1.22-2.80)$ & 0.004 & & \\
\hline Caregiving problems, yes & $2.59(1.68-3.99)$ & $<0.001$ & & \\
\hline \multicolumn{5}{|l|}{$\begin{array}{l}\text { Index of COVID-19-related stressful events } \\
\text { No events }\end{array}$} \\
\hline One event & & & $2.45(1.27-4.72)$ & 0.007 \\
\hline Two events & & & $9.56(5.44-16.80)$ & $<0.001$ \\
\hline Three events & & & $16.64(9.08-30.51)$ & $<0.001$ \\
\hline Four or more events & & & $17.81(9.92-31.97)$ & $<0.001$ \\
\hline
\end{tabular}




\section{Discussion}

The results of study 1 showed that elevated risk of serious mental illness was associated with disruption in social and occupational COVID-19-related stressful events, but not with those more educational aspects. As more COVID-19-related stressful events were generated, the risk of serious mental illness increased, suggesting the risk of overwhelming an individual's psychological capacities during times of enhanced stress. ${ }^{9}$ These COVID-19related stressful events have important implications for daily functioning and preserving psychological well-being. In terms of social relationships, an inability to seek help outside the home means that COVID-19 has been associated with increased domestic problems. ${ }^{10}$ In the context of the UK, it is estimated that the current gross domestic product will shrink by $9 \%$ and around 7.6 million jobs will be lost. Roughly $24 \%$ of the UK labour force are at risk because of COVID-19-related lockdowns. ${ }^{11}$ Those with the lowest income are the most vulnerable. $^{12}$ This may suggest that the most vulnerable groups are prone to exhibit elevated serious mental illness when compared with less vulnerable groups.

The results pattern of study 2 were similar to those in study 1 , albeit with different COVID-19-related stressful events. Elevated serious mental illness and probable ICD-11 adjustment disorder were associated with participants' own health and caregiving problems and, to a lesser extent, housing problems and the health problems of a loved one. In addition, the model associated with elevated serious mental illness was found to be more robust than the model associated with the modified ICD-11 adjustment disorder. This may be because the $\mathrm{K} 6$ scale is capturing more aspects of anxiety and depression.

Cumulative stressful events take their medical toll. This has been found in the association between cumulative stress and disrupted hypothalamic-pituitary-adrenocortical axis functioning, ${ }^{13}$ lower oxytocin level in pregnant women ${ }^{14}$ and Takotsubo cardiomyopathy. ${ }^{15}$ This adds to the known detrimental effect of psychological stress on health (e.g. immunoregulatory balance) and the association with inflammatory disease, ${ }^{16}$ cardio vascular disease, ${ }^{17}$ immune system $^{18}$ and immune inflammatory reactions. ${ }^{19}$ Psychological stress also takes an economic toll directly and indirectly, and increases the burden on the health system. ${ }^{20}$

The results of study 2 support a dose-response model of stress and post-traumatic stress disorder; ${ }^{21}$ the more COVID-19-related stressful events were experienced by the participants, the more the mental toll, evidenced in an increased likelihood of probable ICD11 adjustment disorder. The dose-response association of cumulative disruptions in COVID-19-related stressful events or cumulative COVID-19-related stressful events on probable ICD-11 adjustment disorder echoes previous findings. ${ }^{22,23}$

From a clinical point of view, these disruptions and COVID-19related stressful events are important, as they can readily lead to an excessive burden on the general population. Clinicians may expect to see more referrals based on resource loss and any ineffective coping associated with this. Another consequence of the COVID-19 pandemic is that populations generally at lower risk of mental disorder before COVID-19 may become at risk because of the disruption to their everyday lives. ${ }^{24-26}$ Our findings suggest that clinicians should be aware of disruption in COVID-19-related stressful events as a source of serious mental illness, especially when there is risk of a 'storm' that overwhelms an individual's capacity to cope. Although the association between a medical cytokine storm and health outcomes from COVID-19 disease ${ }^{27,28}$ has recently been questioned, ${ }^{29}$ COVID-19 is a global stressful event, with salient psychosocial consequence.

\section{General limitations and conclusions}

We recognise several study limitations. Our study was crosssectional and, although we recruited respondents from a wide range of ages, our responses were self-reported. In addition, we used two unrelated samples recruited at different time points. This is a weakness when compared with a longitudinal study. We also report relatively low response rates $(37 \%$ and $42 \%$, respectively), although these response rates are similar to other large internet surveys. ${ }^{30}$ We had no information on past psychological conditions. We did not consider additional psychological consequences of anxiety, such as stereotyping and prejudice, reported during the SARS ${ }^{31}$ and COVID-19 pandemics. ${ }^{32,33}$ Another potential bias is the possibility that people who are distressed about COVID-19 may be more likely to fill out a survey, thus inflating the strength of the relationship.

The cost of psychological stress is known to be associated with medical conditions and illnesses, ${ }^{34}$ and increased risk for mortality. ${ }^{21}$ Psychiatrists should be aware that certain COVID-19-related stressful events can lead to serious psychological problems. ${ }^{24}$ They need to pay particular attention to patients who report cumulative COVID-19related stressful events, and consider them for mental health assessment and treatment.

In sum, to our knowledge, this is first study to empirically examine the association between COVID-19-related stressful events and cumulative COVID-19-related stressful events, with an elevated risk of serious mental illness at two different time points. Moreover, we report a novel association between single COVID19-related stressful events and cumulative COVID-19-related stressful events with probable ICD-11 adjustment disorder.

Future studies should take a longitudinal approach, and use biological markers of stress to gain a better insight into the health costs of experiencing specific and cumulative COVID-19-related stressful events.

Menachem Ben-Ezra (D), School of Social Work, Ariel University, Israel; WaiKai Hou (DD, Department of Psychology, Centre for Psychosocial Health, The Education University of Hong Kong, Hong Kong, China; Robin Goodwin (D), Department of Psychology, University of Warwick, UK

Correspondence: Menachem Ben-Ezra. Email: menbe@ariel.ac.il

First received 7 Aug 2020, final revision 21 Nov 2020, accepted 26 Nov 2020

\section{Data availability}

The data that support the findings of this study are available from the corresponding author M.B.-E., upon reasonable request.

\section{Author contributions}

M.B.-E. collected data, performed the statistical analysis and drafted the initial manuscript. M.B.-E., W.K.H. and R.G. provided the concept and design of study and were involved in the analysis. M.B.-E., W.K.H. and R.G. were involved in interpretation of data. W.K.H. and R.G. revised the manuscript for important intellectual content. All authors read and approved the final manuscript.

\section{Funding}

M.B.-E. was supported by Ariel's University Research Authority internal funding (grant RA2000000106).

\section{Declaration of interest}

None. 


\section{References}

1 Ball T, Wace C, Smyth C, Brown D. Hunt for contacts of Coronavirus-stricken pair in York. The Times, 2020 (https://www.thetimes.co.uk/article/hunt-forcontacts-of-coronavirus-stricken-pair-in-york-dh363qf8k).

2 Lyons K. Coronavirus latest: 23 March at a glance. The Guardian, 2020 (https:// www.theguardian.com/politics/live/2020/mar/23/uk-coronavirus-live-newslatest-boris-johnson-minister-condemns-people-ignoring-two-metre-distance-rule-in-parks-as-very-selfish)

3 Ben-Ezra M, Sun S, Hou WK, Goodwin R. The association of being in quarantine and related COVID-19 recommended and non-recommended behaviors with psychological distress in Chinese population. J Affect Disord 2020; 275: 66-8.

4 Hobfoll SE. Conservation of resources: a new attempt at conceptualizing stress. Am Psychol 1989; 44: 513-24.

5 Hou WK, Lai FTT, Ben-Ezra M, Goodwin R. Regularizing daily routines for mental health during and after the COVID-19 pandemic. J Glob Health 2020; 10 : 020315.

6 Kessler RC, Barker PR, Colpe LJ, Epstein JF, Gfroerer JC, Hiripi E, et al. Screening for serious mental illness in the general population. Arch Gen Psychiatry 2003: 60: $184-9$.

7 Shevlin $M$, Hyland $P$, Ben-Ezra $M$, Karatzias $T$, Cloitre $M$, Vallières $F$, et al. Measuring ICD-11 adjustment disorder: the development and initial validation of the International Adjustment Disorder Questionnaire. Acta Psychiatr Scand 2020; 141: 265-74.

8 Steiger JH. Tests for comparing elements of a correlation matrix. Psychol Bull 1980; 87: 245-51.

9 Holmes EA, O'Connor RC, Perry HV, Tracey I, Wessely S, Arseneault L, et al. Multidisciplinary research priorities for the COVID-19 pandemic: a call for action for mental health science. Lancet Psychiatry 2020; 7: 547-60.

10 Townsend M. Revealed: surge in domestic violence during Covid-19 crisis. The Guardian, 2020 (https://www.theguardian.com/society/2020/apr/12/domesticviolence-surges-seven-hundred-per-cent-uk-coronavirus).

11 Gant J. Lockdown will cost a quarter of UK jobs as 6.5 million positions are to be axed with the hospitality industry set to be crippled by coronavirus crisis, research says. Mail Online, 2020 (https://www.dailymail.co.uk/news/article8234081/Lockdown-cost-QUARTER-UK-jobs-6-5-MILLION-positions-axed.html).

12 Allas T, Canal M, Hunt V. COVID-19 in the United Kingdom: Assessing Jobs at Risk and the Impact on People and Places. McKinsey \& Company, 2020 (https://www.mckinsey.com/industries/public-sector/our-insights/covid-19in-the-united-kingdom-assessing-jobs-at-risk-and-the-impact-on-people-andplaces).

13 Suglia SF, Staudenmayer J, Cohen S, Enlow MB, Rich-Edwards JW, Wright R Cumulative stress and cortisol disruption among Black and Hispanic pregnant women in an urban cohort. Psychol Trauma 2010; 2: 326-34.

14 Samuel S, Hayton B, Gold I, Feeley N, Carter CS, Zelkowitz P. Attachment security and recent stressful life events predict oxytocin levels: a pilot study of preg nant women with high levels of cumulative psychosocial adversity. Attach Hum Dev 2015; 17: 272-87.

15 Rosman L, Dunsiger S, Salmoirago-Blotcher E. Cumulative impact of stressful life events on the development of Takotsubo cardiomyopathy. Ann Behav Med 2017; 51: 925-30
16 Marshall GD. The adverse effects of psychological stress on immunoregulatory balance: applications to human inflammatory diseases. Immunol Allergy Clin North Am 2011; 31: 133-40.

17 Brotman DJ, Golden SH, Wittstein IS. The cardiovascular toll of stress. Lancet 2007; 370: 1089-100

18 Gu HF, Tang CK, Yang YZ. Psychological stress, immune response, and atherosclerosis. Atherosclerosis 2012; 223: 69-77.

19 Iwata M, Ota KT, Duman RS. The inflammasome: pathways linking psychological stress, depression, and systemic illnesses. Brain Behav Immun 2013; 31: 105-14.

$20 \mathrm{Kalia}$ M. Assessing the economic impact of stress - the modern day hidden epidemic. Metabolism 2002; 51S: 49-53.

21 Dohrenwend BS, Dohrenwend BP. Stressful Life Events: Their Nature and Effects. John Wiley and Sons, 1974.

22 Kaysen D, Rosen G, Bowman M, Resick PA. Duration of exposure and the doseresponse model of PTSD. J Interpers Violence 2010; 25(1): 63-74.

23 Hamer M, Kivimaki M, Stamatakis E, Batty GD. Psychological distress and infectious disease mortality in the general population. Brain Behav Immun 2019; 76 : 280-3.

24 Marsh S. Fifth of vulnerable people considered self-harm in UK lockdown. The Guardian, 2020 (https://www.theguardian.com/society/2020/jul/12/vulnerable-people-self-harm-suicide-uk-lockdown-coronavirus?).

25 Goodwin R, Sugiyama K, Sun S, Aida J, Ben-Ezra M. Psychological distress after the Great East Japan Earthquake: two multilevel 6-year prospective analyses. Br J Psychiatry 2020; 216: 144-50.

26 Hou WK, Liu H, Liang L, Ho J, Kim H, Seong E, et al. Everyday life experiences and mental health among conflict-affected forced migrants: a meta-analysis. J Affect Disord 2020; 264: 50-68.

27 Jose RJ, Manuel A. COVID-19 cytokine storm: the interplay between inflammation and coagulation. Lancet Respir Med 2020; 8: e46-7.

28 Zhou F, Yu T, Du R, Fan G, Liu Y, Liu Z, et al. Clinical course and risk factors for mortality of adult inpatients with COVID-19 in Wuhan, China: a retrospective cohort study. Lancet 2020; 395: 1054-62.

29 Sinha, P, Matthay, MA, Calfee, CS. Is a "cytokine storm" relevant to CoVID-19? JAMA Intern Med 2020; 180(9): 1152-4.

30 Ben-Ezra M, Hyland P, Karatzias T, Maercker A, Hamama-Raz Y, Lavenda O, et al. A cross-country psychiatric screening of ICD-11 disorders specifically associated with stress in Kenya, Nigeria and Ghana. Eur J Psychotraumatol 2020; 11: 1720972

31 Washer P. Representations of SARS in the British newspapers. Soc Sci Med 2004; 59: 2561-71.

32 American Psychological Association (APA). Combating Bias and Stigma Related to COVID-19: How to Stop the Xenophobia that's Spreading Along with the Coronavirus. APA, 2020 (https://www.apa.org/topics/covid-19-bias).

33 Brooks SK, Webster RK, Smith LE, Woodland L, Wessely S, Greenberg N, et al. The psychological impact of quarantine and how to reduce it: rapid review of the evidence. Lancet 2020; 395: 912-20.

34 Cohen S, Janicki-Deverts D, Miller GE. Psychological stress and disease. J Am Med AssOC 2007; 298: 1685-7.

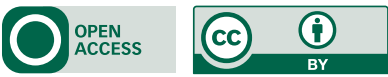

